\title{
Prescriptions hors-AMM : comment en pratique les identifier, les encadrer, informer et les suivre?
}

\author{
Claire Le Jeunne ${ }^{1}$, Nathalie Billon ${ }^{2}$ Anne Dandon ${ }^{3}$ et les participants à la table ronde $N^{\circ} 3$ de Giens XXVIII : \\ Driss Berdai ${ }^{4}$, Yolande Adgibi ${ }^{5}$, Jean-François Bergmann ${ }^{6}$, Régis Bordet ${ }^{7}$, Anne Carpentier ${ }^{8}$, \\ Emmanuelle Cohn ${ }^{9}$, Soizic Courcier ${ }^{10}$, Danièle Girault ${ }^{11}$, Sylvia Goni ${ }^{12}$, Pascale Jolliet ${ }^{13}$, François Liard ${ }^{14}$, \\ Sonia Prot-Labarthe ${ }^{15}$, Tabassome Simon ${ }^{16}$, Christine Vernotte ${ }^{17}$ et Jérémie Westerloppe $e^{18 \dagger}$
}

1 Service de Médecine interne, Assistance Publique - Hôpitaux de Paris, Hôtel Dieu, Paris, France

2 Laboratoire Sanofi, Paris, France

3 Haute Autorité de Santé, Saint-Denis la Plaine, France

4 Centre hospitalier de Bordeaux, Bordeaux, France

5 Orphandev, Centre hospitalier de Marseille, Marseille, France

6 Centre hospitalier Lariboisière, Paris, France

7 Pharmacologie, Faculté de Médecine, Lille, France

8 Les entreprises du médicaments (LEEM), Paris, France

9 Haute Autorité de Santé, Saint-Denis la Plaine, France

10 Laboratoire GlaxoSmithKline, Marly-le-Roi, France

11 Laboratoire Novartis Pharma, Rueil-Malmaison, France

12 Laboratoire Lundbeck, Issy-les-Moulineaux, France

13 Centre hospitalier de Nantes, Nantes, France

14 Cabinet de médecine générale, Saint-Épain, France

15 Pharmacie Hôpital Robert Debré, Paris, France

16 Centre hospitalier Saint Antoine, Paris, France

17 Laboratoire Lilly France, Suresnes, France

18 Laboratoire Celgene, Paris, France

Texte reçu le 12 avril 2013 ; accepté le 7 mai 2013

\section{Mots clés :}

AMM ;

hors-AMM ;

AMM conditionnelle ; protocole thérapeutique temporaire ;

réglementation ;

recommandations temporaires d'utilisation
Résumé - À la suite de l'affaire Médiator, et de la loi de sécurité sanitaire de décembre 2011, la prescription hors-autorisation de mise sur le marché (AMM) est une réelle préoccupation partagée par tous les acteurs de santé. Le hors-AMM au sens le plus strict est défini par toutes les prescriptions qui ne correspondent pas au résumé des caractéristiques du produit (RCP), en particulier celles qui sont en dehors des indications et des posologies définies par l'AMM, pour des raisons évidentes de sécurité.

Les raisons de la prescription hors-AMM sont diverses, conscientes comme inconscientes, elles ont pour objectif de répondre d'une part aux besoins médicaux non couverts, à ceux des populations peu ou pas étudiées dans les essais mais chez lesquelles une extrapolation de l'AMM est raisonnable (prescriptions de bon sens), et d'autre part à des besoins de santé publique urgents (baclofène, femmes enceintes et médicaments du virus de l'immunodéficience humaine...). Toutes ces prescriptions mériteraient d'être étudiées en vue d'une AMM. Par contre, il existe des prescriptions hors-AMM qu'il faut limiter voire sanctionner quand il s'agit de prescriptions compassionnelles, de complaisance et/ou ne reposant sur aucun fondement scientifique. Les prescriptions hors-AMM ne sont pas faciles à dépister car si le prescripteur est tenu d'écrire la mention « hors-AMM » sur son ordonnance lorsqu'il se livre à ce type de prescription, force est de constater qu'en pratique il ne le fait qu'exceptionnellement. Les pharmaciens qui délivrent le médicament tout comme les caisses d'assurance maladie qui le remboursent, n'ont pas accès au diagnostic (ou l'indication visée) ; il faut donc, pour les identifier, avoir recours au croisement des bases de données à notre disposition (pharmacovigilance, programme de médicalisation des systèmes d'information [PMSI], livret thérapeutique hospitalier, échantillon généraliste des bénéficaires de l'Assurance maladie [EGB] ou système national d'information inter régions [SNIIRAM], données de ventes, d'études de marché...). Le dossier patient informatisé partagé par tous résoudra possiblement cette problématique. Le dispositif de recommandation temporaire d'utilisation (RTU) proposé par la loi de sécurité du médicament ne répondra à cette problématique qu'en partie pour les molécules récemment commercialisées (extension d'indication).

$\dagger$ Les articles, analyses et propositions issus des Ateliers de Giens n'engagent que leurs auteurs et ne préjugent pas de la position de leur organisme de tutelle. 
Ce dispositif dérogatoire et transitoire autorisera une prescription hors-AMM reconnue, possiblement remboursée et surveillée pendant 3 ans. Ces RTU concerneront la faible partie du « hors-AMM » qui dispose de preuves d'une balance bénéficerisque positive (AMM conditionnelle anciennement protocole thérapeutique temporaire [PTT]) mais ceci est loin de viser la majorité des prescriptions hors-AMM. De ce fait et afin d'améliorer le bon usage des médicaments, il apparait important de proposer un système d'encadrement de tout le « hors-AMM » à l'aide d'une commission dédiée : la commission du « hors$\mathbf{A M M} »$ qui permettra de conduire à des recommandations d'utilisation (RU) ou non.

Abréviations : voir en fin d'article.

\section{Introduction}

En 2010, l'affaire Médiator a mis en exergue les risques inhérents à l'utilisation d'un médicament hors du champ de son autorisation de mise sur le marché (AMM). L'utilisation des médicaments hors de leur AMM, qui est une pratique très répandue, est alors devenue un sujet de préoccupation partagé par tous les acteurs de santé. Lors des groupes de réflexions des Assises du médicament un groupe a été spécifiquement dédié à l'encadrement de la prescription hors-AMM. ${ }^{[1]}$

Les recommandations de ce groupe étaient :

1. détecter et suivre les prescriptions « hors-AMM »;

2. détecter les risques de prescription «hors-AMM» dès les essais cliniques et développer la recherche clinique publique ;

3. améliorer la fiabilité des recommandations de prescription « hors-AMM » émises par les autorités sanitaires (Haute autorité de santé (HAS), Institut national du cancer [INCA], Agence française de sécurité sanitaire des produits de santé [Afssaps]...);

4. signaler davantage les prescriptions « hors-AMM » injustifiées, en particulier celles qui sont dangereuses ;

5. renforcer les dispositifs dérogatoires (autorisation temporaire d'utilisation [ATU], protocole thérapeutique temporaire [PTT], « article $56 »)^{[2]}$ en systématisant le suivi des patients et en programmant la sortie du « hors-AMM »;

6. étendre progressivement l'article 56 aux recommandations de prescription « hors-AMM » émises par les autorités sanitaires ;

7. mobiliser la HAS, l'Agence nationale de sécurité du médicament et des produits de santé (ANSM), l'INCA et le Comité économique des produits de santé (CEPS) et assurer leur coordination, sous la responsabilité du Ministère de la santé ;

8. informer les patients et les professionnels de santé ;

9. responsabiliser les acteurs du système de santé.

Ces recommandations majoritairement de bon sens ont été à l'origine de la loi de sécurité sanitaire de décembre 2011 qui précise dans le Code de la santé publique (CSP) les conditions acceptables de prescriptions hors-AMM et la création d'un nouveau dispositif d'encadrement du hors-AMM médicalement justifié, les recommandations temporaires d'utilisation (RTU), dont l'établissement et la diffusion seront centralisées au niveau de l'ANSM. ${ }^{[3]}$

Aujourd'hui tous les textes réglementaires nécessaires à la mise en application de ce dispositif sont publiés. ${ }^{[4-6]}$ Les responsabilités de chaque acteur sont claires, même si quelques points pratiques nécessitent d'être éclaircis. Dans ce contexte il est important de réfléchir à une gestion du hors-AMM tenant compte de ce nouveau dispositif, mais aussi des situations que ce dispositif ne pourra encadrer. Cette question a été l'objet en septembre 2012 d'un des ateliers des rencontres pharmacologiques de Giens. Cet article a comme objectif de présenter les propositions élaborées par ce groupe pour identifier, encadrer, informer et suivre les prescriptions hors-AMM.

\section{Méthodes}

En réunion préparatoire le groupe a adopté une définition consensuelle. À partir de là, il a recensé toutes les situations dans lesquelles il y avait des prescriptions hors-AMM et les moyens possibles et existants pour les identifier. Enfin s'est posée la question des dispositifs mis en place par les autorités de santé pour accompagner le hors-AMM et valider certaines situations de bon sens qui ne feront a priori jamais l'objet d'un dépôt d'AMM.

\section{1. Définition du hors-AMM}

Le groupe est tombé d'accord sur la définition la plus simple et la plus large. Le hors-AMM concerne toutes les prescriptions en dehors du cadre du résumé des caractéristiques du produit (RCP) d'un médicament. Ces situations concernent surtout les rubriques indication et posologie du RCP, mais également les populations particulières pouvant donner lieu à des limitations d'utilisation dans un RCP souvent par manque de données exploratoires. C'est le cas des enfants, des femmes enceintes, des sujets âgés, des faibles ou gros poids, des insuffisants rénaux ou hépatiques, pour lesquels le besoin de prise en charge est évidemment très important. Cette pratique est très répandue : elle porte sur 15 à $20 \%$ du total des prescriptions selon certains auteurs, voire davantage dans des domaines comme la pédiatrie, la gérontologie, la cardiologie, la cancérologie. ${ }^{[1]}$

\subsection{Les raisons de la prescription hors-AMM}

La prescription d'un médicament hors de son AMM est une pratique nécessaire dans certaines situations en l'absence de données au moment de la commercialisation, données qui ne seront que 
très rarement validées par une AMM ultérieure. Malgré un arsenal thérapeutique de plus en plus large et spécifique, trop de situations cliniques restent sans solution thérapeutique validée par une AMM. Dans de telles situations, le prescripteur est amené à s'orienter vers des solutions thérapeutiques sur la base de données pouvant être limitées ou insuffisantes pour l'AMM, mais qu'il considère comme pouvant apporter une réponse thérapeutique suffisante. Dans d'autres situations, des données cliniques disponibles robustes n'ont pas encore donné lieu à une modification de l'AMM. Il peut en effet y avoir un décalage entre l'évolution des connaissances et l'évolution de l'AMM. Enfin, les études réalisées dans un objectif d'enregistrement concernent la plupart du temps des populations générales sélectionnées par des critères d'inclusion stricts, et ne permettent pas d'évaluer le rapport bénéfice/risque d'un médicament pour des populations particulières pour lesquelles les données restent rares (gériatrie, pédiatrie) et qui sont pourtant souvent les principales intéressées. Soixante pour cent des médicaments remboursés le sont à des personnes de plus de 65 ans. ${ }^{\text {[7] }}$

Néanmoins, prescrire hors-AMM est une pratique risquée. Tout d'abord pour le patient compte tenu du rapport bénéfice/risque souvent méconnu particulièrement pour le dénominateur, pour le médecin qui engage sa responsabilité, pour le laboratoire pharmaceutique qui doit répondre à ce hors-AMM sans qu'il en connaisse toujours l'existence, et enfin pour l'Assurance maladie, pour laquelle le risque devient économique puisque le médicament prescrit horsAMM sera remboursé sans que ceci soit forcément justifié et dans une population qui souvent n'était pas incluse dans la population cible initiale.

\subsection{Quels pourraient être les systèmes d'identification et de suivi du hors-AMM?}

Les sources d'identifications des prescriptions hors-AMM sont multiples mais non structurées. Les prescriptions hors-AMM ne sont pas faciles à dépister car si le prescripteur est tenu d'écrire la mention « hors-AMM » sur son ordonnance lorsqu'il se livre à ce type de prescription, force est aujourd'hui de constater qu'en pratique il ne le fait qu' exceptionnellement. Les pharmaciens qui délivrent le médicament tout comme les caisses d'Assurance maladie qui le remboursent n'ont pas accès au diagnostic (ou l'indication visée) ; il faut donc, pour les identifier, avoir recours au croisement des bases de données disponibles. Il peut s'agir d'outils de suivi des cas de pharmacovigilance, des bases de données hospitalières suivant la gestion des médicaments ou les remboursements au sein des pharmacies à usage intérieur, des données de vente ou d'études de marché des laboratoires (volume par rapport à la population cible initialement prévue), ou encore des études en conditions réelles d'utilisation et également les données fournies malgré tout par les caisses d'Assurance maladie. Toutefois, ces différentes sources sont souvent indépendantes et non partagées. Leur croisement serait nécessaire pour obtenir une information complète et représentative, c'est aujourd' hui complexe. En revanche, une veille bibliographique peut à partir de sources publiques mettre en exergue des pratiques hors-AMM. Certaines utilisations hors-AMM peuvent également émerger avec des problèmes de santé publique médiatisés obligeant à regarder de près ces pratiques médicales. Ces pratiques devraient être davantage regardées et analysées. Mais quelle que soit la source, en plus de la prescription, il est indispensable de pouvoir identifier les diagnostics. Le dossier patient informatisé pourrait être une solution (malheureusement ce n'est pas à l'ordre du jour).

\subsection{Les dispositifs existants en 2012 pour accompagner le hors-AMM}

L'article R4127-8 du CSP reconnait depuis 1971, la liberté de prescription des médecins qui doivent prendre en compte les avantages, les inconvénients et les conséquences de leurs prescriptions que ce soit pour les examens complémentaires ou les médicaments.

La mention : «prescription hors-AMM » est alors indispensable et entraîne le non remboursement du médicament.

Avant la loi du 29 décembre $2011^{[3]}$ l'encadrement du horsAMM était représenté par 2 dispositifs :

1. Les référentiels nationaux de bon usage des médicaments de la liste hors groupe homogène de séjour (hors-GHS) [PTT] élaborés par les autorités de santé (ANSM, HAS et INCA) avec l'aide d'experts après une analyse scientifique de la littérature et évaluation du rapport bénéfice-risque dans des situations non prévues par l'AMM. Ce dispositif permet en cas de présence sur cette liste du médicament de justifier la prise en charge financière par l'Assurance maladie (médicaments coûteux, maladies rares rendant les inclusions dans les essais thérapeutiques difficiles, absence d'alternatives thérapeutiques). Ce dispositif va se terminer fin juin 2014.

2. Le deuxième dispositif est le remboursement dérogatoire dit Article 56 (L162-17-2-1 du CSS) qui autorise la prise en charge dérogatoire et temporaire des médicaments, produits ou prestations par l'assurance maladie, après évaluation par la HAS et l'ANSM. Il concerne des médicaments utilisés hors du panier de biens et services remboursables pour la prise en charge d'affections « longue durée » ou de maladies rares et ce en l'absence d'alternative (repose sur des recommandations d'experts). Il peut être assorti ou non d'une demande de suivi des patients et d'un dépôt d'AMM. Pour le moment ce dispositif persiste

\section{Résultats et propositions du groupe}

\subsection{Typologie du hors-AMM}

Les prescriptions hors-AMM sont plus ou moins justifiées selon le besoin thérapeutique. Elles reposent sur des niveaux de preuves 
Tableau I. Typologie des différentes réponses à envisager lors de hors-AMM observé.

\begin{tabular}{|c|c|c|}
\hline Niveau de preuve $B / R$ favorable & Besoin / Problème identifié & Réponse au besoin \\
\hline Existence de preuves & Non ou mal couvert & Mérite l'AMM \\
\hline Ébauche de preuves & $\begin{array}{l}\text { Non ou mal couvert } \\
\text { Mais principe de précaution }\end{array}$ & Besoin de consolider les preuves \\
\hline Extrapolation avec bon sens de l'AMM & $\begin{array}{l}\text { AMM validée seulement dans des populations nor- } \\
\text { mées } \\
\text { AMM dans un autre pays }\end{array}$ & $\begin{array}{l}\text { Extrapoler l'AMM avec validité dans des populations } \\
\text { particulières notamment maladies rares et maladies } \\
\text { pédiatriques AMM de bon sens }\end{array}$ \\
\hline Pas de preuves & $\begin{array}{l}\text { Attitude compassionnelle. } \\
\text { Requête (inappropriée) de réponse médicamenteuse } \\
\text { Véritable mésusage (mauvais usage) } \\
\text { Expérimentation sauvage } \\
\text { Identification de nouvelles pistes thérapeutiques }\end{array}$ & $\begin{array}{l}\text { Initier l'évaluation } \\
\text { Sanctions }\end{array}$ \\
\hline
\end{tabular}

AMM : autorisation de mise sur le marché ; B/R : bénéfice risque.

Tableau II. RTU : avantages et inconvénients.

\begin{tabular}{l|l}
\hline Avantages & Inconvénients \\
\hline $\begin{array}{l}\text { Encadre le hors-AMM pour un médicament ayant déjà une AMM } \\
\text { Assure un B/R favorable }\end{array}$ & $\begin{array}{l}\text { Mais qu'une partie du hors-AMM } \\
\text { Temps dévolu au recueil des données trop court (3 ans) } \\
\text { Des alerteurs oubliés : industriels, société savantes } \\
\text { À préciser }\end{array}$ \\
Elargit les bases de l'alerte & $\begin{array}{l}\text { La difficulté de revendiquer une AMM, au niveau national, sur la base } \\
\text { de données de niveau de preuve correct. } \\
\text { Euro-compatible pour des AMM européennes. } \\
\text { Maintien de quelques interrogations sur les modalités de définition de } \\
\text { la convention } \\
\text { Interrogation sur les modalités du recueil des données }\end{array}$ \\
\hline
\end{tabular}

AMM : autorisation de mise sur le marché ; B/R : bénéfice risque ; RTU : recommandation temporaire d'utilisation.

très variables selon les situations. Nous proposons dans le tableau I d'établir une typologie théorique mais pragmatique des différentes réponses à donner face à une prescription hors-AMM, en fonction de son niveau de preuve et du besoin médical existant.

\subsection{Une première réponse pour encadrer le hors-AMM justifié : les recommandations temporaires d'utilisation et des propositions d'évolution}

Défini dans la réforme du médicament de décembre 2011, il s'agit d'un dispositif d'encadrement du hors-AMM justifié, mais ce sous certaines conditions. Les RTU concernent des spécialités pharmaceutiques ayant déjà une AMM mais dans une autre indication. Elles ne sont possibles qu'en absence d'alternative médicamenteuse appropriée disposant d'une AMM ou d'une ATU. Ces
RTU sont élaborées par l'ANSM et s'accompagnent d'un recueil de données obligatoire permettant d'évaluer et de suivre l'efficacité, les effets indésirables et les conditions réelles d'utilisation. Elles sont associées à une convention signée entre l'ANSM et le(s) laboratoire(s) concerné(s) avec un possible engagement du titulaire de déposer à terme une demande d'AMM. ${ }^{[8]}$

Ce nouveau dispositif présente à la fois des avantages et des inconvénients (tableau II) dont deux paraissent particulièrement limitatifs : la durée de la période limitée à 3 ans est trop courte pour pouvoir collecter via un essai clinique des données permettant de déposer une AMM et l'eurocompatibilité d'une telle mesure (transposabilité dans les autres pays européens).

Un troisième problème est celui du laboratoire qui devrait déposer une demande d'extension d'AMM pour une thérapeutique déjà génériquée ; des procédures facilitatrices pourraient voir le jour comme des procédures allégées pour un génériqueur, voire une 
Tableau III. Propositions pour gérer le hors-AMM.

\begin{tabular}{|c|c|c|c|}
\hline Niveau de preuve $B / R$ favorable & Besoin / Problème identifié & Réponse au besoin & Propositions \\
\hline Existence de preuves & Non ou mal couvert & Mérite l'AMM & $\begin{array}{l}\text { RTU concomitante à la validation de } \\
\text { l'AMM par un dossier bibliographique }\end{array}$ \\
\hline Ébauche de preuves & $\begin{array}{l}\text { Non ou mal couvert } \\
\text { principe de précaution }\end{array}$ & $\begin{array}{l}\text { Besoin de consolider les } \\
\text { preuves }\end{array}$ & $\begin{array}{l}\text { Essais cliniques + } \\
\text { RTU }\end{array}$ \\
\hline $\begin{array}{l}\text { Extrapolation avec bon sens de } \\
\text { l'AMM }\end{array}$ & $\begin{array}{l}\text { AMM validée seulement dans des } \\
\text { populations normées } \\
\text { AMM dans un autre pays }\end{array}$ & $\begin{array}{l}\text { Extrapoler l'AMM avec } \\
\text { validité dans des populations } \\
\text { particulières notamment } \\
\text { maladies rares et maladies } \\
\text { pédiatriques }\end{array}$ & $\begin{array}{l}\text { Création de RU, dispositif plus souple } \\
\text { Liberté de prescription remboursée }\end{array}$ \\
\hline Pas de preuves & $\begin{array}{l}\text { Attitude compassionnelle } \\
\text { Requête (inappropriée) de réponse } \\
\text { médicamenteuse } \\
\text { Véritable mésusage (mauvais usage) } \\
\text { Expérimentation sauvage } \\
\text { Identification de nouvelles pistes } \\
\text { thérapeutiques }\end{array}$ & Initier l’évaluation & $\begin{array}{l}\text { De l'éducation à la sanction } \\
\text { Proposer une autre attitude thérapeutique } \\
\text { Initier une recherche encadrée }\end{array}$ \\
\hline
\end{tabular}

AMM : autorisation de mise sur le marché ; RTU : recommandation temporaire d'utilisation ; RU : recommandation d'utilisation.

AMM académique. Les propositions permettant de gérer le horsAMM pourraient être différentes selon le niveau de preuves du bénéfice/risque $(\mathrm{B} / \mathrm{R})$ et l'existence d'alternatives thérapeutiques dans la pathologie étudiée (tableau III).

1. Dans le cas idéal, l'existence de preuves d'un rapport bénéfice/risque favorable doit permettre le dépôt d'un dossier d'enregistrement, la RTU permettant durant les 3 ans la mise à disposition du produit de façon encadrée dans l'attente de la validation de l'autorisation de mise sur le marché.

2. Dans de nombreux cas, la période de 3 ans de la RTU ne permettra pas d'obtenir de données suffisantes pour déposer une demande d'AMM. La création de RTU renouvelable permettrait alors de générer suffisamment de données pour conduire à l'AMM.

3. Si ce nouveau délai n'était toujours pas suffisant et que le besoin médical reste non satisfait, une recommandation d'utilisation (RU) pourrait être élaborée. Ces RU concerneraient de petites populations, et/ou d'anciennes molécules pour lesquelles, l'utilisation serait validée par les sociétés savantes correspondantes sous forme de recommandations au prix déjà existant (procédure peu différente d'un article 56)

Les RU s'accompagneraient d'un suivi patient dont il faudrait évidemment préciser les objectifs. La prescription pourrait faire l'objet d'un circuit particulier avec une ordonnance dédiée. Cette RU pourrait être réévaluée tous les 5 ans. Comme les RTU, ce dispositif ne pourrait bien sûr être efficace et fiable sans une communication appropriée des prescripteurs concernés, des pharmaciens et des patients (figure 1).
Lorsque les données du rapport bénéfice/risque ont été collectées dans une population générale et ont permis une autorisation d'AMM, dans une population suffisamment large, l'aide de modèles mathématiques pourrait permettre des données d'extrapolation pharmacocinétiques dans ces populations restreintes et une recommandation d'utilisation « de bon sens » devrait voir le jour et permettrait de contrôler cette prescription hors-AMM sans la lourdeur d'un nouveau dépôt d'AMM.

4. Enfin en l'absence d'ébauche de preuves, il faut soit bannir le mésusage (les actions à mettre en place dans ce cas pourront aller de l'éducation à la sanction en passant par le contrôle), soit initier la mise en évidence de preuve d'un bénéfice/risque favorable notamment dans des domaines de santé publique et ce en l'absence d'alternatives, cette initiative pouvant être portée par des fonds publics (programme hospitalier de recherche clinique [PHRC] ou autres appels d'offres).

\subsection{Nécessité de surveiller et d'encadrer tout le hors-AMM : une commission du hors-AMM}

Comme nous venons de le voir, avec les RTU, l'ANSM s'est dotée d'un dispositif efficace pour encadrer une utilisation horsAMM médicalement justifiée et soutenue par des données relativement solides. Toutefois ce dispositif a ses limites et ne répondra pas à l'encadrement de toutes les prescriptions hors-AMM. Une commission du hors-AMM pourrait être créée pour prendre en compte la globalité des situations hors-AMM. Elle serait constituée de 


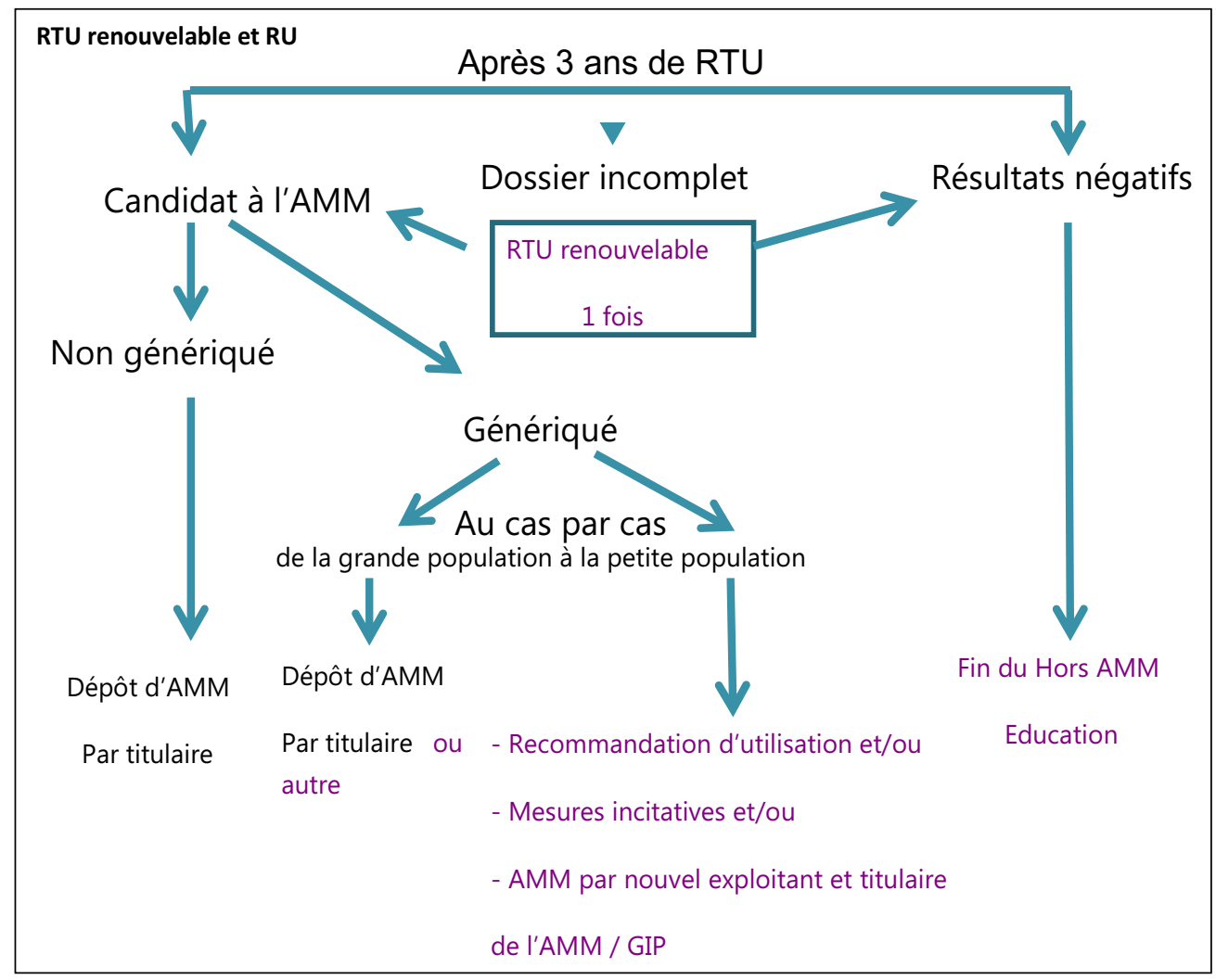

Fig. 1. Circuit proposé de RTU et RU.

AMM : autorisation de mise sur le marché ; GIP : groupement d'intérêt public GIP-CenGEPS ; RTU : recommandation temporaire d'utilisation ; RU : recommandation d'utilisation.

représentants de la HAS, des centres régionaux de pharmacovigilance (CRPV), de la cellule RTU de l'ANSM, de la Direction générale de la santé (DGS), de la Direction de la sécurité sociale (DSS) et de la Caisse nationale d'Assurance maladie (CNAM) [à moyens constants].

Elle assurerait les missions suivantes :

- veille du hors-AMM à partir de l'interrogation et du suivi des bases de données existantes, et d'une veille bibliographique internationale;

- élaboration des protocoles pharmaco-épidémiologiqueséconomiques ;

- recherche de modèles mathématiques de pharmacocinétique de population pour les terrains à risque comme le vieillard et la femme enceinte ;

- mise en place et suivi des RTU ;

- mise en place des dispositifs d'accompagnement des prescriptions hors-AMM dans des indications de santé publique (comme le protocole «consultation d'avis multidisciplinaires de traitements d'exception en addictologie »
[CAMTEA] mis en place dans le nord de la France encadrant l'utilisation du liorésal dans le sevrage alcoolique);

- réalisation d'un état des lieux des molécules anciennes en validant leur niveau de preuve avec le soutien le cas échéant de centres de référence, médicaments génériqués, AMM anciennes très larges sans indication bien définie ;

- éducation et communication vers les différents acteurs par l'intermédiaire de courriers d'informations aux professionnels de santé, de publications sur interne, d'une « newsletter du hors-AMM »...

Cette commission pourrait ainsi établir des recommandations selon les différentes situations identifiées pour inciter à terme à «aller à l'AMM » en fonction du besoin thérapeutique.

\section{Conclusion}

$\mathrm{Au}$ terme d'une réflexion approfondie avec la participation d'acteurs d'horizons diverses, 3 propositions importantes émanent du groupe : la possibilité d'une RTU renouvelable une fois soit 6 ans 
maximum, la possibilité pour certaines molécules dans des situations bien particulières d'obtenir une recommandation d'utilisation (RU) renouvelable tous les 5 ans en l'absence d'alternative et de promoteur porteur du projet, et surtout pour encadrer tous ces dispositifs, la création d' une commission paritaire du hors-AMM qui permettrait de surveiller dans l'intérêt de tous, les prescriptions hors-AMM et d'accorder parfois des extensions d'AMM dites « de bon sens » notamment pour les populations restreintes.

\section{Conflits d'intérêts. Aucun.}

Abréviations. AMM : autorisation de mise sur le marché ; ANSM : Agence nationale de sécurité du médicament et des produits de santé ; ATU : autorisation temporaire d'utilisation ; B/R : bénéfice/ risque ; CEPS : Comité économique des produits de santé ; CRPV : centre régional de pharmacovigilance; CSP : Code de la santé publique ; EGB : échantillon généraliste des bénéficaires de l'Assurance maladie ; HAS : Haute autorité de santé ; INCA : Institut national du cancer ; PHRC : programme hospitalier de recherche clinique; PMSI : programme de médicalisation des systèmes d'information ; PTT : protocole thérapeutique temporaire ; RCP : résumé des caractéristiques du produits; RU : recommandation d'utilisation ; RTU : recommandation temporaire d'utilisation ; SNIIRAM : système national d'information inter-régions.

\section{Références}

1. Rapport de synthèse des Assises du médicament. Groupe 3. http://www.legifrance.gouv.fr

2. Article 56 de la loi de financement de la sécurité sociale. http://www.legifrance.gouv.fr

3. LOI n $^{\circ}$ 2011-2012 du 29 décembre 2011 relative au renforcement de la sécurité sanitaire du médicament et des produits de santé. http://www.legifrance.gouv.fr

4. Décret $n^{\circ} 2012-597$ du 27 avril 2012 relatif à l'Agence nationale de sécurité du médicament et des produits de santé. http://www.legifrance.gouv.fr

5. Décret $\mathrm{n}^{\circ}$ 2012-742 du 9 mai 2012 relatif aux recommandations temporaires d'utilisation des spécialités pharmaceutiques. http://www.legifrance.gouv.fr

6. Décret $\mathrm{n}^{\circ} 2012-740$ du 9 mai 2012 relatif à la prise en charge dérogatoire par l'assurance maladie des spécialités pharmaceutiques bénéficiant d'une recommandation temporaire d'utilisation ou de certains produits et prestations. http://www.legifrance.gouv.fr

7. Laude A. Dans la tourmente du Mediator : prescription hors-AMM et responsabilités. Recueil Dalloz, 27 janvier 2011, n4

8. Emmerich J, Dumarcet N, Lorence A. France's new framework for regulating off-label drug use. N Engl J Med 2012 Oct 4; 367(14): 1279-81

Correspondance et offprints: Claire Le Jeunne, Service de Médecine interne, Assistance Publique - Hôpitaux de Paris, Hôtel Dieu, 1 place du Parvis de Notre Dame, 75004 Paris, France.

E-mail : claire.le-jeunne@htd.aphp.fr 